\title{
GIS-Based Noise and Hearing Loss Screening in Publishing Factory
}

\author{
Hassan Mohammadpour ${ }^{1}$, Seyyed Ali Moussavi Najarkola ${ }^{1,{ }^{*}}$, Mahdi Jalali ${ }^{2}$, Amin Hosseini \\ Asl $^{3}$, Alireza Rahmati ${ }^{1}$ \\ ${ }^{1}$ Department of Occupational Hygiene Engineering, Collage of Health, Shahid Beheshti University of Medical Sciences, Tehran, IR Iran \\ ${ }^{2}$ Department of Occupational Hygiene Engineering, Hamadan University of Medical Sciences, Hamedan, IR Iran \\ ${ }^{3}$ Department of Surveying Engineering, Faculty of Civil Engineering, Toosi University of Technology, Tehran, IR Iran \\ ${ }^{*}$ Corresponding author: Seyyed Ali Moussavi Najarkola, Department of Occupational Hygiene Engineering, Collage of Health, Shahid Beheshti University of Medical Sciences, P. O \\ Box:19835-35511, Tehran, IR Iran. Tel: +98-2122432040-41, Fax: +98-2122432037, E-mail: mosavi58@gmail.com.
}

Received: July 20, 2013; Revised: September 1, 2013; Accepted: September 5, 2013

Background: In many industries, noise is attributed as the most prevalent harmful agent threatening workers' health. Geographic Information System(GIS) is an applicable surveying tool in civil engineering. But, the use of GIS for noise and hearing loss screening seems to be partly a new approach.

Objectives: This study aimed to apply GIS in noise and hearing loss screening in the production hall of a publishing industry.

Patients and Methods: A cross-sectional study was conducted on 46 employees working in the production hall of a publishing industry in Iran. First, workers' hearing threshold was examined by Pure-Tone Audiometry(PTA)at 250-8000 Hz frequencies. Then, the production hall area was divided into squares $\left(6 \times 6 \mathrm{~m}^{2}\right)$, taking sound pressure levels (SPL) in each square center by a sound level meter(SLM) according to ISO-9612; 2009. Noise and hearing loss maps were drawn, by Arc GIS-9.2, for different areas of the production hall.

Results: Noise measurements revealed that total sound pressure levels (SPL) in the production hall ranged from 72.3 to $94.5 \mathrm{dBA}$. From the total area of production hall, $20 \%$ of it was found to be exceeding the threshold limit value (SPL $\geq 85 \mathrm{dBA}$ ) on the GIS-prepared noise map. Fisher exact test showed a significant difference between hearing losses ( $\mathrm{HL} \geq 25 \mathrm{~dB}$ ) in the danger zone (SPL $\geq 85 \mathrm{~dB}$ ) and the warning zone $(85>\mathrm{SPL} \geq 65 \mathrm{~dB})(\mathrm{P}=0.005)$. Among the workers, $50 \%$ of them were in the danger zone and $8.8 \%$ of those who were in warning zone had varying degrees of hearing losses.

Conclusions: GIS can play an important role in better noise and hearing loss screening through providing a set of facilities such as: converting point data to area ones, possibility of classification in different domains of prepared area data, generation of reports on map, graphic, and table, etc.

Keywords: Noise; Geographic Information System; GIS; Hearing Loss; Publishing Industry

\section{Introduction}

Nowadays, noise is considered as the most prevalent harmful agent and one of the most common physical factors in many industrial workplaces and environments that can threaten workers' health (1). Generally, no industry is found to be free of noise pollution $(2,3)$. Noise-induced damages have been reported as top 10 hazardous agents-inducing damages in workplaces $(4,5)$. Thereby, it is estimated that 600 million people worldwide are exposed to excessive noise in their workplace which can threaten their health (6). Noise exposure is accompanied by a plenty of complications such as hearing loss, cardiovascular diseases, hypertension, increased risk of death, serious physiological effects, headache, anxiety, and nausea (7-9). The most important effect of noise is hearing damage, the so-called noise-induced hearing loss (NIHL).
NIHL is referred as the most common potentially preventable form of sensory-neural hearing impairment in industrial locations (workplaces) (10). Exposure to excessive noise can induce temporary or permanent damage to the auditory system (10). Temporary threshold shift (TTS) occurs when a short-term exposure to loud noise within 24 hours to 48 hours takes place, which is reversible, but permanent threshold shift (PTS) occurs when prolonged exposure to excessive noise takes place, which causes gradual damages to the cochlear hair cells of the inner ear (10). Some reports estimate that around $30 \mathrm{mil}-$ lion workers in the United States of America are exposed to noise levels above $85 \mathrm{~dB}$ in workplaces (11), and about 10 million people suffer noise-induced hearing loss (NIHL $>25 \mathrm{~dB}$ ) (12). However, there is no precise statistics available on the amount of Iranian workers' actual exposure to excessive noise at industrial workplaces. Other effects the prepared area data in different domains, the generation of reports on map, graphic, and table, etc.

Copyright (C) 2013, Health promotion research center. This is an open-access article distributed under the terms of the Creative Commons Attribution License, which permits unrestricted use, distribution, and reproduction in any medium, provided the original work is properly cited. 
of noise are interference with communication, altered performance, annoyance, distraction, and interference with work or relaxation and physiological responses such as elevated blood pressure and sleep disturbances (10). Therefore, noise can have serious effects on the workers' social and occupational life leading to leaving the job (13). Thus, one can easily find that the critical dimension of the problem is somewhat significant(3). In many countries, noise is classified as the most significantly occupational risk factor in the publishing industry. Results of a cross-sectional study conducted on 274 workers working in 34 publishing companies in the United States of America (USA) showed that $43 \%$ of the workers were exposed to noise levels above $85 \mathrm{dBA}$ SPL during eight working hours (14). Information about an investigation performed on 33 publishing companies in Singapore revealed that the workers are subjected to noise levels ranging from 85to 96 dBA SPL (15). Geographic information system (GIS) is a useful surveying tool for storing, managing, and analyzing the collected data with spatial and descriptive dependencies (16). GIS also examines various statuses and predicts new scenarios through processing data gained by the computer software (16). Ko et al.(2011) used the GIS to draw noise maps and determine the noise impact assessment on hearing system in a study conducted on the citizens of Chungju city (17). Kluijver et al.(2003) used the GIS-based noise map in order to improve the productivity and the quality of noise effect studies (18). Obviously, the use of GIS in different studies regarding the assessment of the effects of noise pollution in Iranian industries can be a new approach and toward progression in acoustic engineering and audiology sciences, especially in occupational hygiene. Publishing industry is a typical industry that involves noisy machines and devices needing to be controlled accurately. For this, the determination of the main noise sources, showing their layout and sound pressure levels (SPLs) on noise map, and the percentage of hearing loss distribution on noise maps are new administrative ideas in occupational health and medicine for making decision in future HSE planning. GIS is also one of the most applicable screening tools that seem to be useful for drawing noise maps in industrial environments for better understanding noisy areas. But the use of GIS for screening of areas associated with hearing loss seems to be somewhat a new approach.

\section{Objectives}

This study aimed to use GIS in noise and hearing loss screening in different areas of the publishing industry for better preventive hearing conservation program (HCP) and setting the worker-to-job fitness plan in nearly future.

\section{Patients and Methods}

A cross-sectional type descriptive-analytical study was conducted on 46 randomly selected workers occupying the production hall of Osveh publishing industry (located in Qom city, the center of Iran) as one of the biggest national publishing industries located in Tehran, Iran. First, all participants were asked to complete a questionnaire with questions about age, job history, head trauma, exposure to noise, mercury, carbon disulfide, carbon monoxide, smoking, and the use of ototoxic drugs, etc. Along with this procedure, workers' hearing threshold was examined by Pure-tone audiometey (PTA) type Amplivox; Model 270 (Germany) at 250-8000 Hz frequencies. Thereby, the employees' hearing loss was examined through air-conduction (AC) audiometry for both left and right ears exactly 48-hours after leaving the job to determine the permanent threshold shifts (PTS). All AC-PTA examinations were tested by an expert and trained audiologist with 10 years of experience, assigned through a private audiology company for this purposes. All hearing examinations were measured in a standard acoustic room that truly met OSHA's audiometric test room requirements. The permanent threshold losses (as the indicators of NIHL) for both ears (average hearing losses in $\mathrm{dB}$ in four frequencies of 500 , $1000,2000,4000 \mathrm{~Hz}$ ) were calculated among workers of production hall dividing into four categories: safe or no damage to hearing system (NIHL $<25)$, minor damage to hearing system $(26<\mathrm{NIHL}<40)$, intermediate damage to hearing system $(41<\mathrm{NIHL}<60)$, and finally, high damage to hearing system (NIHL $>60)(19)$. Sound measurement method (according to ISO-9612; 2009) was followed for noise measurement and consequently, drawing noise maps in production hall (20). Along with, the production hall area was divided into approximately sized squares $\left(6 \times 6 \mathrm{~m}^{2}\right)$ by means of a tape meter, taking a reading in each square center (center of any grid). A Sound Level Meter (SLM; Model CEL-241; manufactured by CEL company, made in England) was used for the measurement of sound pressure levels (SPL). Before any measurement, SLM was calibrated both internally and externally and set in A-frequency weighting network, SLOW mode as RMS detector modes of operation (selected meter response time). A set of measurements including minimum sound pressure level LP,min, maximum sound pressure level LP,max, equivalent sound pressure level (Leq), mean sound pressure level LP,mean, and total pressure levels (SPLT) was recorded made for further purposes. Microphone of the SLM was kept on a distance of 1.5 meters from the ground during whole periods of the measurement process. Noise and hearing loss maps were prepared by Arc GIS-9.2 for different areas of the production hall. Since the collected data have a point nature, the GIS was used for continuously analysis of the collected data. Hence, gridded map of the production hall was initially entered in the GIS, then data related to noise levels (only sound pressure levels as SPL or Lp) were recorded and used in the characteristics table of 
the local layer of the GIS and consequently, for drawing noise map. Kriging interpolation method was used as a proper method for interpolating and converting the point data to continuous area. The map resulted from interpolation allows the possibility of analysis of sound pressure status in different parts of the production hall. Also, with classification of prepared noise map, it is possible to determine and compare the areas of different classes of sound pressure levels. Finally, the sound pressure contours map is obtained from the sound pressure continuous map by use of three-dimensional analysis methods present in the GIS and then, analyzed. It must be stressed that, regarding to distribution of sampled points and possibility study of interpolation in Kriging model, the required interpolation was performed with adequate precision. Briefly, all data used in GIS was originally point ones, then these data were converted to area data with appropriate cell size using interpolation models. Finally, the contours lines were extracted by utilizing the defined modules present in GIS. Coordinates used in this study were local within the production hall area that can be transferred to the global coordinate system as required.

\section{Results}

All participants with the age ranging 22-63 years (42.5 \pm 20.5 years) as well as job history ranging 3-22 years (12.5 \pm 9.5 years) were surveyed to determine the relation between NIHL (and resulted impairment) and noise map (the layout of main noise sources). Other agents affecting noise-induced hearing loss through the exposure to pollutants such as mercury, carbon disulfide, carbon monoxide, smoking, use of ototoxic agents, ototoxic drugs, head trauma, etc. has been shown in Table 1 .

It can be noticed from this table that the highest values for the different pollutants affecting NIHL are related to smoking (34.78\%) and the consumption of ototoxic drugs (6.52\%) only. No significant differences have been found for smoking and the consumption of ototoxic drugs ( $\mathrm{P}>$ 0.05). No significant differences have been obtained for other affecting factors such as mercury, carbon disulfide, carbon monoxide, consumption of ototoxic agents, head trauma $(\mathrm{P}>0.05)$.

\begin{tabular}{llll}
\hline \multicolumn{4}{l}{ Table 1. Different Pollutants Affecting Noise Induced Hearing Loss } \\
\hline Exposure & Yes, No. (\%) & No, No. (\%) & Total, No. (\%) \\
\hline Smoking & $16(34.78)$ & $30(65.22)$ & $46(100)$ \\
\hline Head trauma & $0(0)$ & $46(100)$ & $46(100)$ \\
\hline Ototoxic agents & $0(0)$ & $46(100)$ & $46(100)$ \\
Ototoxic drugs & $3(6.52)$ & $43(93.48)$ & $46(100)$ \\
CO & $0(0)$ & $46(100)$ & $46(100)$ \\
Carbon disulfide & $0(0)$ & $46(100)$ & $46(100)$ \\
Mercury & $0(0)$ & $46(100)$ & $46(100)$ \\
\hline
\end{tabular}

Schematic diagram of noise sources layout and dimensions of the production hall of the publishing industry has been illustrated in Figure 1. The production hall has a $126 \times 23.55 \mathrm{~m}$ dimensions as well as a noise source concentration close to two longitudinal walls.

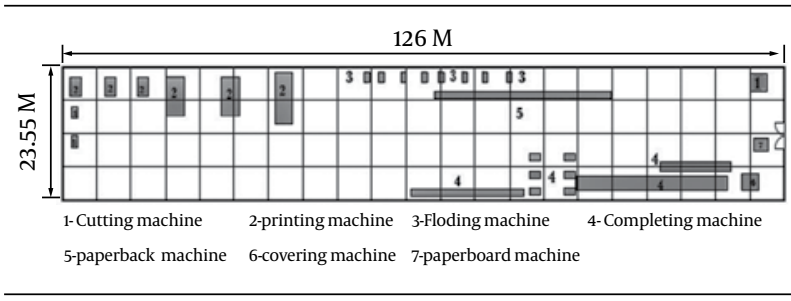

Figure 1. Schematic Diagram of the Production Hall Showing the Location of the Noise Sources

Kriging interpolation-generated noise map of production hall has been shown in Figure 2. It is obvious that the lowest and highest sound pressure values in this noise map were modulated as 72.2 and 94.2 dBA SPL, respectively. Increased intensity of colors in this map shows increased sound pressure levels, so that the red color is the representative of high pressure levels (as a danger zone with SPL $\geq 85 \mathrm{dBA}$ ) and green color is indicator of low pressure levels (as a warning zone with $\mathrm{SPL}<85 \mathrm{dBA}$ ). The results showed that the minimum and maximum sound pressure levels (SPL) at the production hall were 72.3 and 94.5 dBA SPL, respectively. Moreover, the number of stations having measured sound pressure levels (SPL) less and more than threshold limit values-time weighted average (TLV-TWA8-hr $=85 \mathrm{dBASPL}$ ) were 71 and 13 , respectively. The study corresponding to the workers locations in different areas of hall production showed that 13 workers were posed in the danger zone (exposed to: SPL $\geq 85$ $\mathrm{dBA}$ ), while 33 employees were located in the warning zone (subjected to: $85>$ SPL $>65 \mathrm{~dB}$ ) during most of their work time.

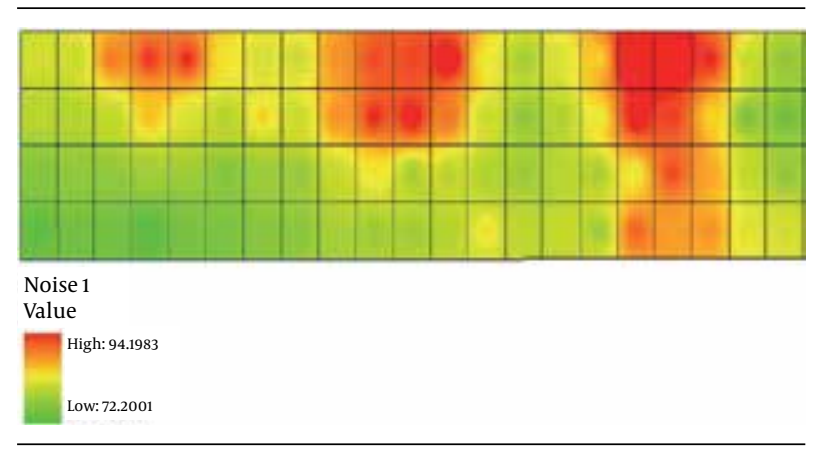

Figure 2. Kriging Interpolation-Generated Noise Map of Production Hall

Sound pressure contour map of the production hall has been demonstrated in Figure 3. Likewise, results obtained from noise measurement and GIS-generated noise maps, especially sound pressure contour map show that devices include: two-color printing machines, four-color 
printing machines, a cutting machine, a folding machine, a drying machine, a sewing machine, and a covering machine respectively play the fundamental role as main noise generating sources in the production hall. As shown in Figure 3, the most areas of the sound pressure counter map is related to $75,78,81,84,87,90$, and $93 \mathrm{dBA}$ respectively.

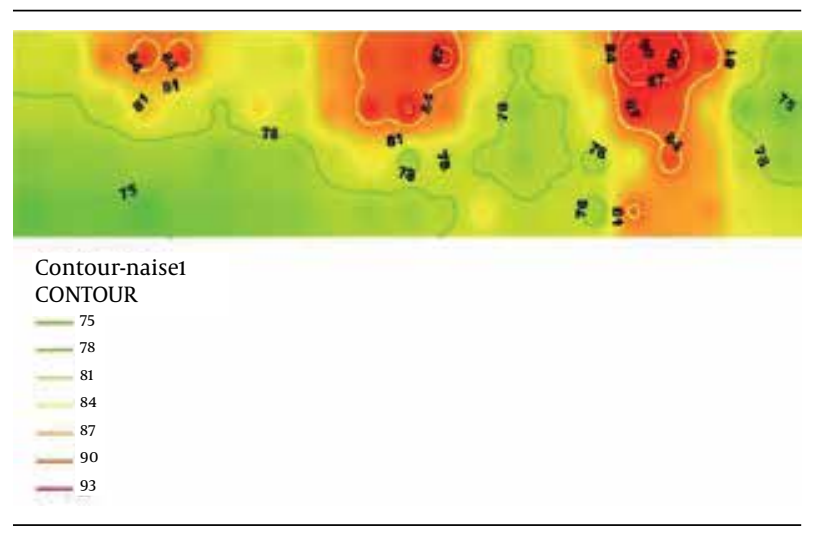

Figure 3. Sound Pressure Contour Map of the Production Hall

The Frequency of damage caused to hearing system in the workers of the production hall (as hearing loss or hearing impairment) has been displayed in Table 2. The mean percentage total permanent threshold losses (as the indicators of NIHL) for both ears (average hearing losses in $\mathrm{dB}$ in four frequencies of 500,1000, 2000, and $4000 \mathrm{~Hz}$ ) among workers of production hall were as follows: $73.9 \%$ had no damages in the hearing system (NIHL $<25), 8.69 \%$ having a minor damage in the hearing system $(26<$ NIHL < 40), and finally, 17.39\% had intermediate damage in the hearing system $(41<\mathrm{NIHL}<60)$. Based on GIS-generated noise map, it has been quantified that almost $20 \%$ of the total area of the production hall had an allowable noise levels (noise levels less than TLV-TWA8$\mathrm{hr}=85 \mathrm{dBA}$ SPL). There is a significant difference between the hearing losses in the danger zone (SPL $\geq 85 \mathrm{dBA}$ ) and warning zone ( $85>\mathrm{SPL}>65 \mathrm{dBA})(\mathrm{P}=0.005)$. Thus, $50 \%$ of workers were located in the danger area, so that $8.8 \%$ of them were suffering from various degrees of hearing losses $(\mathrm{P}<0.05)$.

Table 2. Frequency Distribution of Different Degrees of Hearing Loss in the Workers of the Production Hall

\begin{tabular}{llll}
\hline \multicolumn{3}{c}{ Degree of Hearing Impairment } & Total \\
\hline slight & Intermediate & No damage & \\
\hline $\mathbf{4}$ & 8 & 34 & 46 \\
\hline
\end{tabular}

\section{Discussion}

This study shows that there are several main noise sources in the production hall that play an important role in generating noise levels exceeding the permissible
TLV-TWA8-hr. These noise sources are printing machines (two - color), printing machines (four - color), cutting machines, folding machines and covering machines. A study conducted on noise in publishing industry indicates that excessive noise pollution problem is a serious health and safety problem which requires to be controlled. The minimum and maximum sound pressure levels in the production hall of the publishing industry ranged from 72.3 to $94.5 \mathrm{dBA}$. These findings are consistent with other studies carried out in publishing industries (21-23). A study conducted by Morata et al. in a publishing industry showed that measured sound pressure levels ranged between 71 and $93 \mathrm{~dB}$, indicating noise problem in such industries that should be controlled truly (21). In a study performed in 33 publishing companies in Singapore, Gerges et al. found that the average sound pressure level was around $89 \mathrm{~dB}(15)$. The results obtained from this study showed that most of the workers engaged in publishing industry and had significant hearing damage, were working in the danger zone determined by the GIS (SPL $\geq 85 \mathrm{~dB}$ ) (15). The reason for this high hearing loss in the publishing industry can be attributed to those buildings where the sound pressure levels exceed $85 \mathrm{~dB}$, which can cause damage to the cochlea of the inner hair cells (IHCs) and outer hair cells (OHCs). Moussavi-Najarkola et al. found that the exposure to loud noise led to very high vacuolation and intensive cell injury with the type of hydropic degeneration in outer hair cells (OHCs), mild to moderately pyknotic inner hair cells (IHCs), swollen supportive cells (SC), and slightly thickened basilar membrane (BM) (10, 22). Moussavi-Najarkola et al. believe that the most probable reason for cochlear OHCs' dysfunction damage to the organ of corti can be attributed to the oxidative stress mechanism (22). They reported that metabolic damage occur when toxic waste products, the so-called free radicals (FRs), including reactive oxygen species (ROS) and reactive nitrogen species (RNS), is created after affecting noise stress on the cochlear cells, that induce as sensory-neural hearing loss (SNHL), generally known "noise-induced hearing loss (NIHL)" $(10,22)$. Like the results obtained from this study, NIHL may be caused by a little exposure to high-intensity noise or prolonged exposure to low noise levels. Risks of NIHL are related to the personal susceptibility to noise, the duration and intensity of the exposure to noise $(10,22)$. Therefore, considering the preventive and protective measures such as hearing screening and hearing conservation program (HCP) is preferred for those industries, since NIHL cannot be treated due to irreversible nature of hearing damages caused by excessive noise. As no any other comprehensive study was performed regarding GIS-based screening of noise and hearing loss in the publishing industry, authors suggest further detailed and extensive studies with larger sample size and considering parameters that can affect the noise-induced hearing loss (NIHL), such as demographic factors, exposure to solvents, hyperten- 
sion, etc. Also, the results of frequency analysis as well as dosimetery can be added to the GIS-created noise maps for better understanding of the relation between these factors and noise-induced hearing losses in the workers of publishing industry. Unfortunately, since there is no similar study using GIS in noise study in the publishing industry, so comparing the results with other studies in this field was not possible.

The utilization of hearing conservation programs (HCP) as well as annually hearing screening, seem to be required in industrial locations and occupations that are exposed to excessive noise (24). The elements of this program include: workplace noise exposure monitoring, engineering controls and administrative controls, the use of hearing protection equipment, periodically audiometric testing, and training $(24,25)$. According to the results gained from this research, it can be found that GIS technology can play an important role in noise screening through the utilization of very high technology interpolation (such as Kriging interpolation method) and so forth. It can be deduced that the publishing industry is one of the industries in which the possibility of hearing loss and other effects caused by noise exposure is prevalent. Therefore, the success of this study is evident for necessity to set up further comprehensive studies by the use of GIS for screening noise and HL in the publishing industry and other similar noisy industries.

Conclusions: GIS can play an important role in better noise and hearing loss screening through providing a set of facilities such as: converting point data to area ones, the possibility of classification in different domains of prepared area data, the generation of reports on map, graphic, and table, etc. GIS-created noise maps give a valuable help for the better understanding of the rationale relation between the measured sound pressure levels and examined noise-induced hearing losses in workers occupying different areas of the publishing factory.

\section{Acknowledgements}

The authors would like to thank all employees of the publishing industry and all of them which made the great efforts that this project to be appropriately done.

\section{Authors' Contribution}

Hassan Mohammadpour worked on this study, analyzed the results and wrote the primary draft of this manuscript, Seyyed Ali Moussavi-Najarkola designed the study, contributed in doing the study, and revised the draft manuscript as a supervisor and corresponding author; Mahdi Jalali, Amin Hosseini-asl contributed to data entry in GIS and drawing the noise and hearing loss map; and finally, Alireza Rahmati contributed in setting the manuscript as well as doing the study.

\section{Financial Disclosure}

There is no conflict of interest.

\section{Funding/Support}

There is no support for this study.

\section{References}

1. Ferrite S, Santana V. Joint effects of smoking, noise exposure and age on hearing loss. Occ Med. 2005;55(1):48-53.

2. Mirmohammadi J, Baba Haji Meibodi F, Nourani F. Investigating the hearing tolerance in the workers of the tile factory complex of Meybod. J ShahidSadoughi Univ Med Sci. 2008;16(1):8-13.

3. Zare M, Nasiri P, Shahtaheri S, Golbabaei F, Agha M. Noise Pollution And Hearing Loss In One Of The Oil Industries In Iran. Med J Hormozgan Univ. 2007.

4. Ohinata Y, Yamasoba T, Schacht J, Miller JM. Glutathione limits noise-induced hearing loss. Hearing Res. 2000;146(1):28-34.

5. karlidag T, Yalsin S, Ozturk A. The role of free 94ersia radical in noise induced hearing loss. Auris Nasus Larynx. 2002;94:147-52.

6. Alberti PW. Noise, the most ubiquitous pollutant. Noise and Health. 1998;1(1):3.

7. Abbate C, Concetto G, Fortunato M, Brecciaroli R, Tringali MA, Beninato $\mathrm{G}$, et al. Influence of environmental factors on the evolution of industrial noise-induced hearing loss. Environ Monitor Assess. 2005;107(1-3):351-61.

8. Backstrom T, Mattsson B. Correlation of symptoms in premenstrual tension to oestrogen and progesterone concentrations in blood plasma. A preliminary study. Neuropsychobiology. 1975;1(2):80-6.

9. Stansfeld SA, Matheson MP. Noise pollution: non-auditory effects on health. British Med Bull. 2003;68(1):243-57.

10. Moussavi-Najarkola SA, Khavanin A, Mirzaei R, Salehnia M, Muhammadnejad A, Akbari M. Noise-induced Outer Hair Cells' Dysfunction and Cochlear Damage in Rabbits. Iran Red Crescent Med J. 2012;14(10):647.

11. John LH. Occupational Safety and Health Administration.: U.S. Department of Labor; 2002.

12. Sataloff RT, Sataloff J. Occupational hearing loss.: CRC Press; 2006.

13. Centers for Disease Control and Prevention. Work Related Hearing Loss. Atlanta, USA: NIOSH Publications and Products; 2001. Available from: http://www.cdc.gov/niosh/docs/2001-103/.

14. McMAHON KJ, McMANUS PE. Occupational noise exposure in the printing industry. America Ind Hyg Assoc J. 1988;49(1):34-7.

15. Gerges N, Sehrndt GA, Parthey W. Occupational exposure to noise: Evaluation, prevention and control. 2001. Available from: http:// www.who.int/occupational_health/publications/noise.

16. Assessment of physical agents (noise, heat and illumination) in a hospital of the Society Security Organization in Tehran province. 4 th ed. Hamadan-Iran; 2004.

17. Ko JH, Chang SI, Lee BC. Noise impact assessment by utilizing noise map and GIS: A case study in the city of Chungju, Republic of Korea. Applied Acoustics. 2011;72(8):544-50.

18. De Kluijver H, Stoter J. Noise mapping and GIS: optimising quality and efficiency of noise effect studies. Comput Environ Urban Syst. 2003;27(1):85-102.

19. Meyer JD, Mccunney RJ. Occupational exposure to noise. 4th ed.USA: Lippinctt-Raven; 2007.

20. Acoustics: Determination of occupational noise exposure and estimation of noise-induced hearing impairment.: Int Organ Stand Geneva; 1990.

21. Morata TC, Fiorini AC, Fischer FM, Colacioppo S, Wallingford KM, Krieg EF, et al. Toluene-induced hearing loss among rotogravure printing workers. Scand Journal of work, Environ \& Health. 1997:28998.

22. Moussavi-Najarkola SA, Khavanin A, Mirzaei R, Salehnia M, Muhammadnejad A, Akbari M. Temporary and permanent level shifts in distortion product otoacoustic emissions following noise expo- 


\section{Mohammadpour Het al.}

sure in an animal model. Int J Occ and Environ Med. 2012;3(3 July).

23. Warring RH, Atherley G. Handbook of noise and vibration control.: Trade and technical Press; 1970.

24. Chen G, Liu Y. Mechanisms of noise-induced hearing loss potentia- tion by hypoxia. Hear Res. 2005;200(1):1-9.

25. Chen Z, Ulfendahl M, Ruan R, Tan L, Duan M. Protection of auditory function against noise trauma with local caroverine administration in guinea pigs. Hear Res. 2004;197(1):131-36. 